\title{
CONDITIONED STABLE LÉVY PROCESSES AND THE LAMPERTI REPRESENTATION
}

\author{
M. E. CABALLERO, ${ }^{*}$ Universidad Nacional Autónoma de México \\ L. CHAUMONT, ${ }^{* *}$ Université Pierre et Marie Curie
}

\begin{abstract}
By variously killing a stable Lévy process when it leaves the positive half-line, conditioning it to stay positive, and conditioning it to hit 0 continuously, we obtain three different, positive, self-similar Markov processes which illustrate the three classes described by Lamperti (1972). For each of these processes, we explicitly compute the infinitesimal generator and from this deduce the characteristics of the underlying Lévy process in the Lamperti representation. The proof of this result bears on the behaviour at time 0 of stable Lévy processes before their first passage time across level 0 , which we describe here. As an application, for a certain class of Lévy processes we give the law of the minimum before an independent exponential time. This provides the explicit form of the spatial Wiener-Hopf factor at a particular point and the value of the ruin probability for this class of Lévy processes.
\end{abstract}

Keywords: Positive, self-similar Markov process; Lamperti representation; infinitesimal generator; stable Lévy process conditioned to stay positive; stable Lévy process; conditioning to hit 0 continuously

2000 Mathematics Subject Classification: Primary 60G18; 60G51; 60B52

\section{Introduction and preliminary results}

The stochastic processes which are considered in this work take their values in the Skorokhod space $\mathscr{D}$ of càdlàg trajectories (those that are continuous from the right with left limits). We define this set as follows: $\Delta:=\infty$ being the cemetery point, a function $\omega:[0, \infty) \rightarrow \mathbb{R} \cup \Delta$ belongs to $\mathscr{D}$ if and only if

- for all $t \geq \zeta(\omega), \omega_{t}=\Delta$, where $\zeta(\omega):=\inf \left\{t: \omega_{t}=\Delta\right\}$ is the lifetime of $\omega \in \mathscr{D}$ and $\inf \varnothing=\infty$;

- for all $t \geq 0, \lim _{s \downarrow t} \omega_{s}=\omega_{t}$; and

- for all $t \in(0, \zeta(\omega)), \lim _{s \uparrow t} \omega_{s}:=w_{t-}$ is a finite real value.

The space $\mathscr{D}$ is endowed with the Skorokhod's $J_{1}$ topology. We denote by $X: \mathscr{D} \rightarrow \mathscr{D}$ the canonical process of the coordinates and by $\left(\mathcal{F}_{t}\right)$ the natural Borel filtration generated by $X$, i.e. $\mathcal{F}_{t}=\sigma\left(X_{s}, s \leq t\right)$. A probability measure $\mathrm{P}_{x}$ on $\mathscr{D}$ is the law of a Lévy process if $\left(X, \mathrm{P}_{x}\right)$ starts from $x$, i.e. $\mathrm{P}_{x}\left(X_{0}=x\right)=1$, and has independent and homogeneous increments. Note that $\left(X, \mathrm{P}_{x}\right)=\left(x+X, \mathrm{P}_{0}\right)$ and that the lifetime of $\left(X, \mathrm{P}_{x}\right)$ is either almost surely (a.s.) infinite

Received 30 March 2006; revision received 31 August 2006.

* Postal address: Instituto de Matemáticas, Universidad Nacional Autónoma de México, Mexico 04510 DF.

Email address: emilia@servidor.unam.mx

** Postal address: Laboratoire de Probabilités et Modèles Aléatoires, Université Pierre et Marie Curie, 4 Place Jussieu, 75252 Paris Cedex 05, France. Email address: chaumont@ccr.jussieu.fr 
or a.s. finite. It is well known that, for any Lévy process $\left(X, \mathrm{P}_{x}\right)$ with finite lifetime $\zeta(X)$, there exists a Lévy process $\left(X^{\prime}, \mathrm{P}_{x}\right)$ with infinite lifetime such that, under $\mathrm{P}_{x}$, the random variable $\zeta(X)$ is exponentially distributed and independent of $X^{\prime}$ and, $\mathrm{P}_{x}$-a.s., $X_{t}=X_{t}^{\prime}$ if $t<\zeta(X)$. Furthermore, the parameter of the law of $\zeta(X)$ under $\mathrm{P}_{x}$ does not depend on $x$.

A positive $\left(\mathbb{R}_{+}\right.$-valued), self-similar Markov process (PSSMP) $\left(X, \mathbb{P}_{x}\right), x>0$, is a strong Markov process with paths in $\mathscr{D}$ which possesses a scaling property: there exists a constant $\alpha>0$ such that, for any $b>0$,

$$
\text { the law of }\left(b X_{b^{-\alpha} t}, t \geq 0\right) \text { under } \mathbb{P}_{x} \text { is } \mathbb{P}_{b x} .
$$

These processes are much involved in many areas of probability theory. For instance, the continuous-state branching process obtained as the weak limit of a rescaled discrete branching process is a PSSMP which is associated to a self-similar Lévy tree; see [9]. These processes also appear in fragmentation theory: the mass process of a self-similar fragmentation process is itself a PSSMP [2]. The PSSMPs that we are going to study here have recently been obtained in [5] as limits of rescaled random walks whose laws are in the domain of attraction of a stable law, after they are conditioned to stay positive or conditioned to hit 0 at a finite time; see Sections 3.2 and 3.3, below.

According to Lamperti [10], the set of PSSMPs splits into three exhaustive classes which can be distinguished from each other by comparing their first hitting times at 0 , namely $S=$ $\inf \left\{t>0: X_{t}=0\right\}$. This classification may be summarized as follows.

- $\mathcal{C}_{1}$ is the class of PSSMPs such that $S=\infty, \mathbb{P}_{x}$-a.s. for all starting points $x>0$.

- $\mathcal{C}_{2}$ is the class of PSSMPs for which $S<\infty$ and $X_{S-}=0, \mathbb{P}_{x}$-a.s. for all starting points $x>0$. Processes of this class hit the level 0 in a continuous way.

- $\mathcal{C}_{3}$ is the class of PSSMPs for which $S<\infty$ and $X_{S-}>0, \mathbb{P}_{x}$-a.s. for all starting points $x>0$. These processes hit 0 through a negative jump.

The main result of [10] asserts that any PSSMP may, up to its first hitting time at 0 , be expressed as the exponential of a Lévy process, time changed by the inverse of its exponential functional. Then the underlying Lévy process in the so-called Lamperti representation of $\left(X, \mathbb{P}_{x}\right)$ possesses specific features depending on the class to which $\left(X, \mathbb{P}_{x}\right)$ belongs. More formally, let $\left(X, \mathbb{P}_{x}\right)$ be a PSSMP with index $\alpha>0$ starting from $x>0$, and write the canonical process $X$ in the following form, where, for $t<S, \tau(t)=\inf \left\{s \geq 0: \int_{0}^{s} \exp \left(\alpha \xi_{u}\right) \mathrm{d} u \geq t\right\}$ :

$$
X_{t}=x \exp \left(\xi_{\tau\left(t x^{-\alpha}\right)}\right), \quad 0 \leq t<S .
$$

Then, under $\mathbb{P}_{x}, \xi=\left(\xi_{t}, t \geq 0\right)$ is a Lévy process starting at 0 whose law does not depend on $x>0$ and such that

- if $\left(X, \mathbb{P}_{x}\right) \in \mathcal{C}_{1}$ then $\zeta(\xi)=\infty$ and $\lim \sup _{t \rightarrow \infty} \xi_{t}=\infty, \mathbb{P}_{x}$-a.s.;

- if $\left(X, \mathbb{P}_{x}\right) \in \mathcal{C}_{2}$ then $\zeta(\xi)=\infty$ and $\lim _{t \rightarrow \infty} \xi_{t}=-\infty, \mathbb{P}_{x}$-a.s.; and

- if $\left(X, \mathbb{P}_{x}\right) \in \mathcal{C}_{3}$ then $\zeta(\xi)<\infty, \mathbb{P}_{x}$-a.s.

Note that, for any $t<\int_{0}^{\infty} \exp \left(\alpha \xi_{s}\right) \mathrm{d} s$,

$$
\tau(t)=\int_{0}^{x^{\alpha} t} \frac{\mathrm{d} s}{X_{s}^{\alpha}}, \quad \mathbb{P}_{x} \text {-a.s. },
$$


so (1) is invertible and yields a one-to-one relation between the class of PSSMPs killed at time $S$ and one of the Lévy processes.

Now we recall another important result of [10], which gives the explicit form of the generator of any PSSMP in terms of its underlying Lévy process. Let $\left(X, \mathbb{P}_{x}\right)$ and $\xi$ be any processes related as in (1). We will denote by $\mathcal{K}$ and $\mathcal{L}$ their respective generators and by $\mathfrak{D}_{\mathcal{K}}$ and $\mathfrak{D}_{\mathscr{L}}$ the respective domains of $\mathcal{K}$ and $\mathcal{L}$. Recall that $\mathfrak{D}_{\mathscr{L}}$ contains all the functions with continuous second derivatives on $[-\infty, \infty]$, and that if $\tilde{f}$ is such a function then $\mathcal{L}$ acts as follows for $x \in \mathbb{R}$, where $a \in \mathbb{R}$ and $\sigma>0$ :

$$
\mathcal{L} \tilde{f}(x)=a \tilde{f}^{\prime}(x)+\frac{\sigma}{2} \tilde{f}^{\prime \prime}(x)+\int_{\mathbb{R}}\left[\tilde{f}(x+y)-\tilde{f}(x)-\tilde{f}^{\prime}(x) l(y)\right] \Pi(\mathrm{d} y)-b \tilde{f}(x) .
$$

The measure $\Pi(\mathrm{d} x)$ is the Lévy measure of $\xi$ on $\mathbb{R}$, such that $\Pi(\{0\})=0$ and $\int(1 \wedge$ $\left.|x|^{2}\right) \Pi(\mathrm{d} x)<\infty$. The function $l(\cdot)$ is a bounded Borel function such that $l(y) \sim y$ as $y \rightarrow 0$. In the last term, $b \geq 0$ corresponds to the killing rate of $\xi$, i.e. the parameter of $\zeta(\xi)$ ( $b=0$ if $\xi$ has infinite lifetime). We use primes to denote differentiation. It is important to note that in (2) the choice of the function $l(\cdot)$ is arbitrary and the coefficient a is the only one which depends on this choice.

Theorem 6.1 of [10] may be stated as follows.

Theorem 1. ([10].) If $f:[0, \infty] \rightarrow \mathbb{R}$ is such that $f, x f^{\prime}$, and $x^{2} f^{\prime \prime}$ are continuous on $[0, \infty]$, then they belong to the domain, $\mathfrak{D}_{\mathcal{K}}$, of the infinitesimal generator of $\left(X, \mathbb{P}_{x}\right)$, which acts as follows for $x>0$, where $\Theta(\mathrm{d} u)=\Pi(\mathrm{d} u) \circ \log u$ for $u>0$ :

$$
\begin{aligned}
\mathcal{K} f(x)= & \frac{1}{x^{\alpha}} \int_{\mathbb{R}^{+}}\left[f(u x)-f(x)-f^{\prime}(x) l(\log u)\right] \Theta(\mathrm{d} u) \\
& +a x^{1-\alpha} f^{\prime}(x)+\frac{\sigma}{2} x^{2-\alpha} f^{\prime \prime}(x)-b x^{-\alpha} f(x) .
\end{aligned}
$$

This expression determines the law of the process $\left(X_{t}, 0 \leq t \leq S\right)$ under $\mathbb{P}_{x}$.

To present the results of this paper, let us first consider two examples in the continuous case. The first one is when $\left(X, \mathbb{P}_{x}\right)$ is the standard real Brownian motion absorbed at level 0 . The process $\left(X, \mathbb{P}_{x}\right)$ is a PSSMP which belongs to the class $\mathcal{C}_{2}$ and has index $\alpha=2$, and it is well known (see, e.g. [6]) that its associated Lévy process in the Lamperti representation (1) is given by $\xi=\left(B_{t}-t / 2, t \geq 0\right)$, where $B$ is a standard Brownian motion. The second example is when $\left(X, \mathbb{P}_{x}\right)$ is the Brownian motion conditioned to stay positive. This process corresponds to the three-dimensional Bessel process, i.e. the norm of a three-dimensional Brownian motion. Here $\left(X, \mathbb{P}_{x}\right)$ is a PSSMP which belongs to the class $\mathcal{C}_{1}$ and has index $\alpha=2$, and the underlying Lévy process is given by $\xi=\left(B_{t}+t / 2, t \geq 0\right)$.

Similarly, it is possible to obtain PSSMPs from any stable Lévy process $\left(X, \mathrm{P}_{x}\right)$ with index $\alpha \in(0,2)$, through the same operations. More precisely, by killing $\left(X, \mathrm{P}_{x}\right)$ when it enters the negative half-line, i.e. considering

$$
X_{t} \mathbf{1}_{\{t<T\}} \text { with } T=\inf \left\{t \geq 0: X_{t} \leq 0\right\},
$$

we obtain a PSSMP $\left(X, \mathbb{P}_{x}\right)$ which belongs to the class $\mathcal{C}_{2}$ or $\mathcal{C}_{3}$ according respectively to whether or not $\left(X, \mathrm{P}_{x}\right)$ has negative jumps. Also, by conditioning a stable Lévy process to stay positive, i.e. taking

$$
\mathbb{P}_{x}(\cdot)=\lim _{t \rightarrow \infty} \mathrm{P}_{x}(\cdot \mid T>t), \quad x>0,
$$


we obtain a PSSMP $\left(X, \mathbb{P}_{x}\right)$ belonging to $\mathcal{C}_{1}$. We may also give an interpretation for the processes conditioned to hit 0 continuously: such processes belong to $\mathcal{C}_{3}$.

The main goal of this paper is to identify the underlying Lévy process in the Lamperti representation for each of these processes by computing their infinitesimal generators and using Lamperti's result recalled above. This will be done in Section 3. In Section 4 we deduce from the results of Section 3 the law of the minimum achieved before an independent exponential time has elapsed for an important class of Lévy processes. This gives an expression for the Wiener-Hopf factor of these Lévy processes at a particular point, i.e. the law of $\inf _{s \leq e(k)} \xi_{s}$, where $\xi$ is a Lévy process whose characteristics are described in Corollary 1 and $e(k)$ is an independent random variable with a special parameter $k$. We also find the law of the overall minimum for another class of Lévy processes, whose law is given by Corollary 2. This calculation is equivalent to the problem of finding the explicit form of the corresponding ruin probability, which has recently been studied for other classes of Lévy processes by Lewis and Mordecki [11]. The next section is devoted to further preliminary results, the main one of which is of some interest in its own right, independently of the rest of the paper. It extends a result of Bingham [3] and Rivero [12] which describes the asymptotic behaviour of $\mathrm{P}_{x}(T \leq t)$ as $t$ goes to 0 , i.e. the small tail of first passage times of stable Lévy processes.

\section{The small tail of first passage times of stable Lévy processes}

In the remainder of the paper, $\left(X, \mathrm{P}_{x}\right)$ will denote a stable Lévy process with index $\alpha \in(0,2)$ starting at $x \in \mathbb{R}$. Since stable Lévy processes have infinite lifetimes, the characteristic exponent of $\left(X, \mathrm{P}_{x}\right)$ is defined by $\mathrm{E}_{0}\left[\exp \left(\mathrm{i} \lambda X_{t}\right)\right]=\exp (t \psi(\lambda)), t \geq 0, \lambda \in \mathbb{R}$, where

$$
\psi(\lambda)=\mathrm{i} a \lambda+\int_{\mathbb{R}}\left(\mathrm{e}^{\mathrm{i} \lambda y}-1-\mathrm{i} \lambda y \mathbf{1}_{\{|y|<1\}}\right) \nu(y) \mathrm{d} y .
$$

The density of the Lévy measure is

$$
v(y)=c_{+} y^{-\alpha-1} \mathbf{1}_{\{y>0\}}+c_{-}|y|^{-\alpha-1} \mathbf{1}_{\{y<0\}},
$$

where $c_{+}$and $c_{-}$are two nonnegative constants such that $c_{+}+c_{-}>0$. Note also that the constant $a$ is related to $c_{+}, c_{-}$, and $\alpha$ as follows: $a=\left(c_{+}-c_{-}\right) /(1-\alpha), \alpha \neq 1$. In the case where $\alpha=1$, we will suppose that $\left(X, \mathrm{P}_{x}\right)$ is a symmetric Cauchy process, so we have $c_{+}=c_{-}$and $a=0$. We suppose moreover that neither $\left(X, \mathrm{P}_{x}\right)$ nor $\left(-X, \mathrm{P}_{x}\right)$ is a subordinator. We emphasize that, although (3) is valid for any $\alpha \in(0,2)$, when $\alpha<1$ the drift part of this expression may be inserted in the integral, whence the characteristic exponent has the simpler form $\psi(\lambda)=\int_{\mathbb{R}}\left(\mathrm{e}^{\mathrm{i} \lambda y}-1\right) v(y) \mathrm{d} y$.

Recall that the characteristic exponent of $(X, \mathbb{P})$ may be written in the following form for $\alpha \in(0,1) \cup(1,2)$ :

$$
\mathrm{E}_{0}\left[\exp \left(\mathrm{i} \lambda X_{t}\right)\right]=\exp \left(-c t|\lambda|^{\alpha}[1-\mathrm{i} \beta \operatorname{sgn}(\lambda) \tan (\pi \alpha / 2)]\right), \quad \lambda \in \mathbb{R} .
$$

Here

$$
c=-\left(c_{+}+c_{-}\right) \Gamma(-\alpha) \cos \frac{\pi \alpha}{2}, \quad \beta=\frac{c_{+}-c_{-}}{c_{+}+c_{-}},
$$

and we use the notation

$$
\Gamma(-\alpha)= \begin{cases}-\alpha^{-1} \Gamma(1-\alpha) & \text { if } 0<\alpha<1 \\ \alpha^{-1}(\alpha-1)^{-1} \Gamma(2-\alpha) & \text { if } 1<\alpha<2\end{cases}
$$


We refer the reader to [14] or [13, Theorem 14.10 and its proof, pp. 83-85] for more details. It appears from the above expressions for the characteristic exponent that the law of any stable Lévy process may be identified from the three constants $c_{+}, c_{-}$, and $\alpha$.

It has been proved by Bingham [3, Proposition $3 \mathrm{~b}$ and Theorem 4b] and Rivero [12, Section 2.3] that, for any $x>0$,

$$
\lim _{t \downarrow 0} \frac{1}{t} \mathrm{P}_{x}(T \leq t)=\frac{k}{x^{\alpha}},
$$

where the constant $k$ was explicitly computed in [3] and is given by

$$
k=c\left(1+\beta^{2} \tan ^{2} \frac{\pi \alpha}{2}\right)^{1 / 2} \Gamma(\alpha) \frac{\sin (\pi \alpha \rho)}{\pi} .
$$

By definition $\rho:=\mathrm{P}_{0}\left(X_{1}<0\right)$, and it is well known from [14] that this constant has the expression

$$
\rho=\frac{1}{2}-(\pi \alpha)^{-1} \arctan \left(\beta \tan \frac{\pi \alpha}{2}\right) .
$$

Note that we always have $\alpha \rho \leq 1$. Moreover, we can easily check that $\left(X, \mathrm{P}_{x}\right)$ has no negative jumps if and only if one (and, thus, all) of the three following conditions holds:

$$
c_{-}=0 \quad \beta=1 \quad \Longleftrightarrow \alpha \rho=1 \text {. }
$$

For $\alpha=1$, (4) and (6) can be reduced to $\mathrm{E}_{0}\left[\exp \left(\mathrm{i} \lambda X_{t}\right)\right]=\exp \left(-c_{+} \pi t|\lambda|\right)$ and $k=c_{+}=c_{-}$, respectively. (Note that in [3] the value of $k$ was not defined for $\alpha=1$, although this case was not explicitly excluded.) We emphasize that Rivero's result [12] concerns the more general setting of positive, self-similar Markov processes. Also, in the case where $\left(X, \mathrm{P}_{x}\right)$ has no negative jumps, we have $k=0$, but [3, Proposition 3b] gives an explicit expression for the asymptotic behaviour of $\mathrm{P}_{x}(T<t)$ as $t \downarrow 0$.

The next lemma implies and completes [3, Theorem 4b]. In particular, it shows that, when $c_{-}>0, \mathrm{P}_{x}(T \leq t)=\mathrm{P}_{x}\left(\inf _{s \leq t} X_{s} \leq 0\right)$ and $\mathrm{P}_{x}\left(X_{t} \leq 0\right)$ are equivalent as $t$ tends to 0 . Moreover the constant $k$ recalled in (6) may be expressed in a much simpler form. An easy computation using (5), (6), and the identity $-\alpha \Gamma(-\alpha) \Gamma(\alpha)=\pi / \sin \pi \alpha$, for $\alpha \in(0,1) \cup(1,2)$, shows that the two forms coincide, i.e. that $k=c_{-} \alpha^{-1}$.

Lemma 1. For any $x>0$,

$$
\lim _{t \downarrow 0} \frac{1}{t} \mathrm{P}_{x}(T \leq t)=\lim _{t \downarrow 0} \frac{1}{t} \mathrm{P}_{x}\left(X_{t} \leq 0\right)=\frac{c_{-}}{\alpha x^{\alpha}} .
$$

In particular, $\lim _{t \downarrow 0}(1 / t) \mathrm{P}_{x}\left(T \leq t, X_{t} \in(0, \infty)\right)=0$.

Proof. The second equality is a direct consequence of the fact that $(1 / t) \mathrm{P}_{0}\left(X_{t} \in \mathrm{d} z\right)$ converges vaguely to $v(z) \mathrm{d} z$ on $\{z:|z|>K\}$ for every $K>0$, as $t \downarrow 0$; see, e.g. [1, Exercise I.1].

To obtain the first equality, it suffices to show that

$$
\lim _{t \downarrow 0} \frac{1}{t} \mathrm{P}_{x}\left(T \leq t, X_{t} \in(0, \infty)\right)=0 .
$$


To this aim, fix $0<\delta<x$ and write

$$
\mathrm{P}_{x}\left(T \leq t, X_{t} \in(0, \infty)\right)=\mathrm{P}_{x}\left(T \leq t, X_{t} \in(0, \delta]\right)+\mathrm{P}_{x}\left(T \leq t, X_{t}>\delta\right) .
$$

Since

$$
\lim _{t \downarrow 0} \frac{1}{t} \mathrm{P}_{0}\left(X_{t} \in(-x, \delta-x]\right)=\frac{c_{-}}{\alpha}\left((x-\delta)^{-\alpha}-x^{-\alpha}\right),
$$

we deduce for the first term on the right-hand side of (8) the following bound, which tends to 0 as $\delta \downarrow 0$ :

$$
\underset{t \downarrow 0}{\limsup } \frac{1}{t} \mathrm{P}_{x}\left(T \leq t, X_{t} \in(0, \delta]\right) \leq \frac{c_{-}}{\alpha}\left((x-\delta)^{-\alpha}-x^{-\alpha}\right) .
$$

To treat the second term of (8), let us write

$$
\begin{aligned}
\mathrm{P}_{x}\left(T \leq t, X_{t}>\delta\right) & =\int_{-\infty}^{0} \int_{0}^{t} \mathrm{P}_{x}\left(T \in \mathrm{d} s, X_{s} \in \mathrm{d} y\right) \mathrm{P}_{x}\left(X_{t}>\delta \mid T=s, X_{s}=y\right) \\
& =\int_{-\infty}^{0} \int_{0}^{t} \mathrm{P}_{x}\left(T \in \mathrm{d} s, X_{s} \in \mathrm{d} y\right) \mathrm{P}_{y}\left(X_{t-s}>\delta\right)
\end{aligned}
$$

where the second equality follows from the Markov property. Again, since $(1 / t) \mathrm{P}_{0}\left(X_{t} \in \mathrm{d} z\right)$ converges vaguely to $v(z) \mathrm{d} z$ on $\{z:|z|>K\}$ for every $K>0$, there exists a constant $C$ such that, for sufficiently small $t$,

$$
\mathrm{P}_{y}\left(X_{t-s}>\delta\right) \leq \frac{C t}{\delta^{\alpha}} \quad \text { for all } s \in(0, t) \text { and } y \leq 0 .
$$

From (9), we have

$$
\mathrm{P}_{x}\left(T \leq t, X_{t}>\delta\right) \leq \mathrm{P}_{x}(T \leq t) \frac{C t}{\delta^{\alpha}}
$$

which proves that $\lim _{t \downarrow 0}(1 / t) \mathrm{P}_{x}\left(T \leq t, X_{t}>\delta\right)=0$, as required.

This result will be used in Section 3.1 to compute the infinitesimal generator of the killed stable Lévy process.

\section{Killed or conditioned stable processes as PSSMPs}

In this section we compute the characteristics of the underlying Lévy process in the Lamperti representation of a PSSMP $\left(X, \mathbb{P}_{x}\right)$ when this process is either a stable Lévy process which is killed when it first hits the positive half-line (Section 3.1), a stable Lévy process conditioned to stay positive (Section 3.2), or a stable Lévy process conditioned to hit 0 continuously (Section 3.3). If $\left(X, \mathrm{P}_{x}\right)$ is a stable subordinator then it can be considered to be automatically conditioned to stay positive, and in this case the characteristics of the underlying Lévy process are those computed by Lamperti [10, Section 6]. Except in this situation, the cases where $\left(X, \mathrm{P}_{x}\right)$ or $\left(-X, \mathrm{P}_{x}\right)$ is a subordinator are not relevant to this study, so they will be implicitly excluded in the sequel. Also, as already mentioned in the introduction, since our results are well known when $\left(X, \mathrm{P}_{x}\right)$ is the standard Brownian motion, we will always suppose that $\alpha \neq 2$. 


\subsection{The killed process}

In this subsection we suppose that $\left(X, \mathbb{P}_{x}\right), x>0$, is a stable Lévy process with index $\alpha \in(0,2)$ which is killed when it first leaves the positive half-line. To define this process more formally, let $\left(X, \mathrm{P}_{x}\right)$ be a stable Lévy process starting at $x>0$. We use the same notation for the characteristics of $\left(X, \mathrm{P}_{x}\right)$ as in Section 2. Recall that $T=\inf \left\{t \geq 0: X_{t} \leq 0\right\}$; the probability measure $\mathbb{P}_{x}$ is thus the law under $\mathrm{P}_{x}$ of the process

$$
X_{t} \mathbf{1}_{\{t<T\}, \quad t \geq 0 .}
$$

(Note that, rather than the killed process, we could also call $\left(X, \mathbb{P}_{x}\right)$ the initial Lévy process $\left(X, \mathrm{P}_{x}\right)$ absorbed at level 0$)$. It is not difficult to see that the process $\left(X, \mathbb{P}_{x}\right)$ is a positive self-similar Markov process with index $\alpha$ such that $S<\infty \mathbb{P}_{x}$-a.s. Furthermore, if $\left(X, \mathrm{P}_{x}\right)$ has no negative jumps then $\left(X, \mathbb{P}_{x}\right)$ ends continuously at 0 , so it belongs to the class $\mathcal{C}_{2}$. If $\left(X, \mathrm{P}_{x}\right)$ has negative jumps then it is known that it crosses the level 0 for the first time by jumping, so $\left(X, \mathbb{P}_{x}\right)$ ends with a jump at 0 and belongs to the class $\mathcal{C}_{3}$. We will compute the infinitesimal generator of $\left(X, \mathbb{P}_{x}\right)$ and deduce from its expression the law of the underlying Lévy process $\xi$ associated with $\left(X, \mathbb{P}_{x}\right)$ in the Lamperti representation.

Specializing the expression given in the introduction for stable Lévy processes, we obtain the infinitesimal generator $\mathcal{A}$, with domain $\mathfrak{D}_{\mathcal{A}}$, of the process $\left(X, \mathrm{P}_{x}\right)$ :

$$
\mathscr{A} f(x)=a f^{\prime}(x)+\int_{\mathbb{R}}\left[f(x+y)-f(x)-y f^{\prime}(x) \mathbf{1}_{\{|y|<1\}}\right] v(y) \mathrm{d} y, \quad f \in \mathfrak{D}_{\mathcal{A}} .
$$

Here we recall (from the start of Section 2) that $v(y)=c_{+} y^{-\alpha-1} \mathbf{1}_{\{y>0\}}+c_{-}|y|^{-\alpha-1} \mathbf{1}_{\{y<0\}}$ is the density of the Lévy measure and that $c_{-} \geq 0, c_{+} \geq 0$, and $a=\left(c_{+}-c_{-}\right) /(1-\alpha)$ if $\alpha \neq 1$, and $a=0$ and $c_{+}=c_{-}$if $\alpha=1$.

In the sequel we will denote by $\mathcal{K}$ the infinitesimal generator of the killed process $\left(X, \mathbb{P}_{x}\right)$. Note that, since the state space of this process is $[0, \infty)$ and 0 is an absorbing state, the domain of $\mathcal{K}$, which we denote by $\mathfrak{D}_{\mathcal{K}}$, is included in the set $\{f:[0, \infty) \rightarrow \mathbb{R}, f(0)=0\}$. From the expression for the infinitesimal generator $\mathcal{A}$, we can deduce the expression for $\mathcal{K}$, as the following result shows.

Theorem 2. Let $\left(X, \mathbb{P}_{x}\right)$ be the PSSMP defined by (10), and let $\mathcal{K}$ be its generator. Let $f \in \mathfrak{D}_{\mathcal{K}}$ be such that the function $\tilde{f}$ defined on $\mathbb{R}$ by

$$
\tilde{f}(x)= \begin{cases}f(x) & \text { if } x>0 \\ 0 & \text { if } x \leq 0\end{cases}
$$

belongs to $\mathfrak{D}_{\mathcal{A}}$. Then

$$
\mathcal{K} f(x)=\mathcal{A} \tilde{f}(x) \text { for } x>0 \quad \text { and } \quad \mathcal{K} f(0)=0 .
$$

The action of the generator $\mathcal{K}$ can also be written as

$$
\begin{aligned}
\mathcal{K} f(x)= & \int_{\mathbb{R}^{+}} \frac{1}{x^{\alpha}}\left[f(u x)-f(x)-x f^{\prime}(x)(u-1) \mathbf{1}_{\{|u-1|<1\}}\right] v(u-1) \mathrm{d} u \\
& +a x^{1-\alpha} f^{\prime}(x)-c_{-} \alpha^{-1} x^{-\alpha} f(x) .
\end{aligned}
$$

Remark 1. We emphasize that the set of functions which is used in the above statement to describe the generator $\mathcal{K}$ contains at least all functions of the set $\{f:[0, \infty) \rightarrow \mathbb{R}, f(0)=0\}$ such that $\tilde{f} \in \mathcal{C}_{b}^{2}(\mathbb{R})$. 
Proof of Theorem 2. Recall that $T=\inf \left\{t \geq 0: X_{t} \leq 0\right\}$ and $S=\inf \left\{t \geq 0: X_{t}=0\right\}$, and let $f$ be a function defined as in the statement of the theorem. Then note that

$$
\begin{aligned}
\mathbb{E}_{x}\left[f\left(X_{t}\right)\right] & =\mathbb{E}_{x}\left[f\left(X_{t}\right) \mathbf{1}_{\{t<S\}}+f(0) \mathbf{1}_{\{t \geq S\}}\right] \\
& =\mathrm{E}_{x}\left[\tilde{f}\left(X_{t}\right) \mathbf{1}_{\{t<T\}}\right] \\
& =\mathrm{E}_{x}\left[\tilde{f}\left(X_{t}\right)\right]-\mathrm{E}_{x}\left[\tilde{f}\left(X_{t}\right) \mathbf{1}_{\{T \leq t\}}\right] .
\end{aligned}
$$

So, for any $x>0$, the generator of the killed process $\left(X, \mathbb{P}_{x}\right)$ is given by

$$
\begin{aligned}
\mathcal{K} f(x) & =\lim _{t \rightarrow \infty} \frac{1}{t} \mathbb{E}_{x}\left[f\left(X_{t}\right)-f(x)\right] \\
& =\lim _{t \rightarrow 0} \frac{1}{t}\left[\mathrm{E}_{x}\left[\tilde{f}\left(X_{t}\right)\right]-\tilde{f}(x)\right]-\lim _{t \rightarrow 0} \frac{1}{t} \mathrm{E}_{x}\left[\tilde{f}\left(X_{t}\right) \mathbf{1}_{\{T \leq t\}}\right] .
\end{aligned}
$$

However, from Lemma 1 and the fact that $\tilde{f}$ is bounded and vanishes on $\mathbb{R}_{-}$, we have

$$
\lim _{t \rightarrow 0} \frac{1}{t} \mathrm{E}_{x}\left[\tilde{f}\left(X_{t}\right) \mathbf{1}_{\{T \leq t\}}\right]=0 .
$$

This proves our first assertion. (The value of $\mathcal{K} f$ at 0 is easily computed.)

To prove the second assertion of the theorem, write

$$
\mathcal{K} f(x)=a f^{\prime}(x)+\int_{\mathbb{R}}\left[\tilde{f}(x+y)-f(x)-y f^{\prime}(x) \mathbf{1}_{\{|y|<1\}}\right] \nu(y) \mathrm{d} y
$$

and let $I$ denote the integral in this equation. Then make the change of variable $y=x(u-1)$, to obtain

$$
I=\frac{1}{x^{\alpha}} \int_{\mathbb{R}}\left[\tilde{f}(x u)-f(x)-x(u-1) f^{\prime}(x) \mathbf{1}_{\{|x(u-1)|<1\}}\right] v(u-1) \mathrm{d} u .
$$

Now rewrite $I$ in the form

$$
\begin{aligned}
I= & \frac{1}{x^{\alpha}} \int_{u>0}\left[\tilde{f}(x u)-f(x)-x(u-1) f^{\prime}(x) \mathbf{1}_{\{|u-1|<1\}}\right] v(u-1) \mathrm{d} u \\
& +\frac{1}{x^{\alpha}} \int_{u>0}\left[x(u-1) f^{\prime}(x)\left(\mathbf{1}_{\{|u-1|<1\}}-\mathbf{1}_{\{|x(u-1)|<1\}}\right)\right] v(u-1) \mathrm{d} u \\
& +\frac{1}{x^{\alpha}} \int_{u<0}\left[\tilde{f}(x u)-f(x)-x(u-1) f^{\prime}(x) \mathbf{1}_{\{|x(u-1)|<1\}}\right] v(u-1) \mathrm{d} u
\end{aligned}
$$

and respectively denote these integrals $I_{1}, I_{2}$, and $I_{3}$. Integral $I_{1}$ is in a suitable form as it is but $I_{3}$ requires additional calculations:

$$
I_{3}=-\frac{f(x)}{x^{\alpha}} \int_{u<0} v(u-1) \mathrm{d} u-\frac{1}{x^{\alpha}} \int_{u<0} x(u-1) f^{\prime}(x) \mathbf{1}_{\{|x(u-1)|<1\}} v(u-1) \mathrm{d} u .
$$

Now suppose that $\alpha \neq 1$ (the case $\alpha=1$ being much simpler). We may verify (after careful calculations) that the sum of $I_{2}$ and the second term of $I_{3}$ gives

$$
\frac{c_{+}-c_{-}}{1-\alpha}\left(1-x^{\alpha-1}\right) \frac{f^{\prime}(x)}{x^{\alpha-1}}=a f^{\prime}(x)\left(x^{1-\alpha}-1\right),
$$


since $a=\left(c_{+}-c_{-}\right) /(1-\alpha)$. We finally calculate the first term of $I_{3}$ :

$$
-\frac{f(x)}{x^{\alpha}} \int_{u<0} v(u-1) \mathrm{d} u=-\frac{f(x)}{x^{\alpha}} \frac{c_{-}}{\alpha} .
$$

Then, by adding the different parts together, we find that

$$
\mathcal{K} f(x)=\frac{a}{x^{\alpha-1}} f^{\prime}(x)+I_{1}-\frac{f(x)}{x^{\alpha}} \frac{c-}{\alpha},
$$

which ends the proof.

Let $\xi$ be the underlying Lévy process in the Lamperti representation of $\left(X, \mathbb{P}_{x}\right)$, as it is stated in (1). Recall that $\xi$ may have a finite lifetime, so its characteristic exponent $\Phi$ is defined by

$$
\mathbb{E}\left[\exp \left(\mathrm{i} \lambda \xi_{t}\right) \mathbf{1}_{\{t<\zeta(\xi)\}}\right]=\exp (t \Phi(\lambda)), \quad \lambda \in \mathbb{R} .
$$

Using Lamperti's result (recalled in Theorem 1) and Theorem 2, we may now give the explicit form of the generator of $\xi$ in the special setting considered in this subsection.

Corollary 1. Let $\xi$ be the Lévy process in the Lamperti representation (1) of the PSSMP $\left(X, \mathbb{P}_{x}\right)$ defined in (10). The infinitesimal generator, $\mathcal{L}$, of $\xi$, with domain $\mathfrak{D}_{\mathscr{L}}$, is given by

$$
\mathcal{L} f(x)=a f^{\prime}(x)+\int_{\mathbb{R}}\left[f(x+y)-f(x)-f^{\prime}(x)\left(\mathrm{e}^{y}-1\right) \mathbf{1}_{\left\{\left|\mathrm{e}^{y}-1\right|<1\right\}}\right] \pi(y) \mathrm{d} y-c_{-} \alpha^{-1} f(x)
$$

for any $f \in \mathfrak{D}_{\mathfrak{L}}$ and $x \in \mathbb{R}$, where $\pi(y)=\mathrm{e}^{y} v\left(\mathrm{e}^{y}-1\right), y \in \mathbb{R}$. Equivalently, the characteristic exponent of $\xi$ is given by

$$
\Phi(\lambda)=\mathrm{i} a \lambda+\int_{\mathbb{R}}\left[\mathrm{e}^{\mathrm{i} \lambda y}-1-\mathrm{i} \lambda\left(\mathrm{e}^{y}-1\right) \mathbf{1}_{\left\{\left|\mathrm{e}^{y}-1\right|<1\right\}}\right] \pi(y) \mathrm{d} y-c_{-} \alpha^{-1} .
$$

The process $\left(X, \mathbb{P}_{x}\right)$ belongs to the class $\mathcal{C}_{3}$ if $c_{-}>0$ and belongs to the class $\mathcal{C}_{2}$ if $c_{-}=0$. In the first case the Lévy process $\xi$ has a finite lifetime with parameter $c_{-} \alpha^{-1}$, and in the second case has an infinite lifetime.

It is rather unusual to see $l(y)=\left(\mathrm{e}^{y}-1\right) \mathbf{1}_{\left\{\left|\mathrm{e}^{y}-1\right|<1\right\}}$ as the compensating function in the expression of the infinitesimal generator or the characteristic exponent of a Lévy process. However, as noted in the introduction, any function $l$ such that $l(y) \sim y$ as $y \rightarrow 0$ may be chosen, and the more classical function $l(y)=y \mathbf{1}_{\{|y|<1\}}$ would have the effect of replacing the parameter $a$ by one whose expression is rather complicated.

Let us consider the unkilled version of $\xi$, i.e. the Lévy process $\tilde{\xi}$ with characteristic exponent

$$
\tilde{\Phi}(\lambda)=\mathrm{i} a \lambda+\int_{\mathbb{R}}\left[\mathrm{e}^{i \lambda y}-1-\mathrm{i} \lambda\left(\mathrm{e}^{y}-1\right) \mathbf{1}_{\left\{\left|\mathrm{e}^{y}-1\right|<1\right\}}\right] \pi(y) \mathrm{d} y .
$$

A natural question to ask is whether the process $\tilde{\xi}$ oscillates, drifts to $-\infty$, or drifts to $\infty$. Let us show that each of these three behaviours may pertain depending on the relative values of $c_{-}$, $c_{+}$, and $\alpha$. From the expression for $\tilde{\Phi}$, we see that $\tilde{\xi}$ is integrable and that

$$
\begin{aligned}
\mathbb{E}\left[\tilde{\xi}_{1}\right]= & -\mathrm{i} \tilde{\Phi}^{\prime}(0)=a+c_{+}\left(\int_{0}^{\log 2} \frac{\left(1+y-\mathrm{e}^{y}\right) \mathrm{e}^{y}}{\left(\mathrm{e}^{y}-1\right)^{\alpha+1}} \mathrm{~d} y+\int_{\log 2}^{\infty} \frac{y \mathrm{e}^{y}}{\left(\mathrm{e}^{y}-1\right)^{\alpha+1}} \mathrm{~d} y\right) \\
& +c_{-} \int_{-\infty}^{0} \frac{\left(1+y-\mathrm{e}^{y}\right) \mathrm{e}^{y}}{\left(1-\mathrm{e}^{y}\right)^{\alpha+1}} \mathrm{~d} y .
\end{aligned}
$$


(Here $\mathbb{P}$ can be any of the measures $\mathbb{P}_{x}, x>0$ ). On the one hand, it is clear from the classification recalled in the introduction that, when $\left(X, \mathrm{P}_{x}\right)$ has no negative jumps (i.e. $\left.c_{-}=0\right)$, the Lévy process $\tilde{\xi}=\xi$ drifts towards $-\infty$, meaning that

$$
\frac{c_{+}}{1-\alpha}+c_{+}\left(\int_{0}^{\log 2} \frac{\left(1+y-\mathrm{e}^{y}\right) \mathrm{e}^{y}}{\left(\mathrm{e}^{y}-1\right)^{\alpha+1}} \mathrm{~d} y+\int_{\log 2}^{\infty} \frac{y \mathrm{e}^{y}}{\left(\mathrm{e}^{y}-1\right)^{\alpha+1}} \mathrm{~d} y\right)<0
$$

for all $c_{+}>0$ and $\alpha \in(1,2)$. (Recall that, in the spectrally one-sided case, we necessarily have $\alpha \in(1,2)$.) On the other hand, when $\left(X, \mathrm{P}_{x}\right)$ has no positive jumps $\left(c_{+}=0\right)$, it is easy to derive from (11) that, for any fixed $c_{-}>0, \lim _{\alpha \downarrow 1} \mathbb{E}\left[\tilde{\xi}_{1}\right]=\infty$ and $\lim _{\alpha \uparrow 2} \mathbb{E}\left[\tilde{\xi}_{1}\right]=-\infty$. Since $\alpha \mapsto \mathbb{E}\left(\tilde{\xi}_{1}\right)$ is continuous, there are values of $\alpha \in(1,2)$ for which $\tilde{\xi}$ drifts to $-\infty$, oscillates, or drifts to $\infty$. This argument and (12) show that, for all $c_{-}>0$ and $c_{+}>0$, there are values of $\alpha \in(1,2)$ for which $\tilde{\xi}$ drifts to $-\infty$.

\subsection{The process conditioned to stay positive}

We again consider a stable Lévy process $\left(X, \mathrm{P}_{x}\right)$ as defined as in Section 2. Formally, the process $\left(X, \mathrm{P}_{x}\right)$ conditioned to stay positive is an $h$-transform of the killed process defined in Subsection 3.1, i.e.

$$
\mathbb{P}_{x}^{\uparrow}(A)=h^{-1}(x) \mathrm{E}_{x}\left[h\left(X_{t}\right) \mathbf{1}_{A} \mathbf{1}_{\{t<T\}}\right], \quad x>0, t \geq 0, A \in \mathcal{F}_{t},
$$

where $h(x)=x^{\alpha \rho}$. The function $h$ being positive and harmonic for the killed process, (13) defines the law of a strong homogeneous Markov process. Moreover, this process is $(0, \infty)$-valued and it is clear that it inherits the scaling property with index $\alpha$ from $\left(X, \mathrm{P}_{x}\right)$. Hence, $\left(X, \mathbb{P}_{x}^{\uparrow}\right)$ is an example of a PSSMP which belongs to the class $\mathcal{C}_{1}$. The following more intuitive (but no less rigorous) construction of the law $\mathbb{P}_{x}^{\uparrow}$ justifies us in referring to $\left(X, \mathbb{P}_{x}^{\uparrow}\right)$ as the Lévy process $\left(X, \mathrm{P}_{x}\right)$ conditioned to stay positive:

$$
\mathbb{P}_{x}^{\uparrow}(A)=\lim _{t \rightarrow \infty} \mathrm{P}_{x}(A \mid T>t), \quad x>0, t \geq 0, A \in \mathcal{F}_{t}
$$

We refer the reader to [7] for a general account on Lévy processes conditioned to stay positive. In particular, it was proved in [7] that $\left(X, \mathbb{P}_{x}^{\uparrow}\right)$ drifts to $\infty$ as $t$ tends to $\infty$, i.e.

$$
\mathbb{P}_{x}^{\uparrow}\left(\lim _{t \rightarrow \infty} X_{t}=\infty\right)=1
$$

Let us also mention that this conditioning has a discrete-time counterpart for random walks. Let $\mu$ be a law in the domain of attraction of the stable law $\left(X_{1}, \mathrm{P}_{0}\right)$ and let $W^{\uparrow}$ be a random walk with law $\mu$, conditioned to stay positive. Then the process $\left(X, \mathbb{P}_{x}^{\uparrow}\right)$ may be obtained as the limit in law of the process $\left(n^{1 / \alpha} W_{\lfloor n t\rfloor}^{\uparrow}, t \geq 0\right.$ ), as $n$ tends to $\infty$ (where $\lfloor x\rfloor$ denotes the least-integer part of $x$ ). This invariance principle has recently been proved in [5]; see also [4] for the case $\alpha=2$.

Since $\left(X, \mathbb{P}_{x}^{\uparrow}\right)$ is an $h$-transform of the killed process $\left(X, \mathbb{P}_{x}\right)$ defined in the previous subsection, its infinitesimal generator, which we denote by $\mathcal{K}^{\uparrow}$, may be derived from $\mathcal{K}$ as follows:

$$
\mathcal{K}^{\uparrow} f(x)=\frac{1}{h(x)} \mathcal{K}(h f)(x), \quad x>0, f \in \mathfrak{D}_{\mathcal{K} \uparrow} .
$$


From (15) and Theorem 2, for $x>0$ and $f \in \mathfrak{D}_{\mathcal{K} \uparrow}$ we obtain

$$
\begin{aligned}
x^{\alpha} \mathcal{K}^{\uparrow} f(x)= & \frac{1}{x^{\alpha \rho}} \int_{\mathbb{R}^{+}}\left[(h f)(u x)-(h f)(x)-x(h f)^{\prime}(x)(u-1) \mathbf{1}_{\{|u-1|<1\}}\right] v(u-1) \mathrm{d} u \\
& \quad+\operatorname{ax}(h f)^{\prime}(x)-c_{-} \alpha^{-1}(h f)(x) \\
= & \int_{\mathbb{R}^{+}}\left[u^{\alpha \rho} f(u x)-f(x)-\left(\alpha \rho f(x)+x f^{\prime}(x)\right)(u-1) \mathbf{1}_{\{|u-1|<1\}}\right] \nu(u-1) \mathrm{d} u \\
& +a x f^{\prime}(x)+\left(a \alpha \rho-c_{-} \alpha^{-1}\right) f(x) .
\end{aligned}
$$

Let us denote by $J$ the integral in the above equation and define $v^{\uparrow}(u)=u^{\alpha \rho} v(u-1)$. Then

$$
\begin{aligned}
J= & \int_{\mathbb{R}^{+}}\left[f(u x)-u^{-\alpha \rho} f(x)-\left(\alpha \rho f(x)+x f^{\prime}(x)\right) u^{-\alpha \rho}(u-1) \mathbf{1}_{\{|u-1|<1\}}\right] v^{\uparrow}(u) \mathrm{d} u \\
= & \int_{\mathbb{R}^{+}}\left[f(u x)-f(x)-x f^{\prime}(x)(u-1) \mathbf{1}_{\{|u-1|<1\}}\right] v^{\uparrow}(u) \mathrm{d} u \\
& +\int_{\mathbb{R}^{+}}\left[u^{\alpha \rho}-1-\alpha \rho(u-1) \mathbf{1}_{\{|u-1|<1\}}\right] v(u-1) \mathrm{d} u f(x) \\
& +\int_{\mathbb{R}^{+}}\left(u^{\alpha \rho}-1\right)(u-1) \mathbf{1}_{\{|u-1|<1\}} v(u-1) \mathrm{d} u x f^{\prime}(x) .
\end{aligned}
$$

The infinitesimal generator of the process $\left(X, \mathbb{P}_{x}^{\uparrow}\right)$ is then given by

$$
\begin{aligned}
\mathcal{K}^{\uparrow} f(x)= & \frac{1}{x^{\alpha}} \int_{\mathbb{R}^{+}}\left[f(u x)-f(x)-x f^{\prime}(x)(u-1) \mathbf{1}_{\{|u-1|<1\}}\right] v^{\uparrow}(u) \mathrm{d} u \\
& +\left(a+a_{1}\right) x^{1-\alpha} f^{\prime}(x)+\left(a \alpha \rho+a_{2}-c_{-} \alpha^{-1}\right) x^{-\alpha} f(x),
\end{aligned}
$$

where

$$
\begin{aligned}
a_{1}= & c_{+} \int_{0}^{1} \frac{(1+x)^{\alpha \rho}-1}{x^{\alpha}} \mathrm{d} x+c_{-} \int_{0}^{1} \frac{(1-x)^{\alpha \rho}-1}{x^{\alpha}} \mathrm{d} x \\
a_{2}= & c_{+}\left(\int_{0}^{1} \frac{(1+x)^{\alpha \rho}-1-\alpha \rho x}{x^{\alpha+1}} \mathrm{~d} x+\int_{1}^{\infty} \frac{(1+x)^{\alpha \rho}-1}{x^{\alpha+1}} \mathrm{~d} x\right) \\
& +c_{-} \int_{0}^{1} \frac{(1-x)^{\alpha \rho}-1+\alpha \rho x}{x^{\alpha+1}} \mathrm{~d} x,
\end{aligned}
$$

and $a$ and $\rho$ are as given in Section 2 .

Let us compute the constant $a_{2}$ in terms of $\alpha, c_{-}$, and $c_{+}$. When $\alpha<1$, with obvious changes of variables and integration by parts, we have

$$
\begin{aligned}
a_{2} & =c_{+}\left(\int_{0}^{\infty} \frac{(1+x)^{\alpha \rho}-1}{x^{\alpha+1}} \mathrm{~d} x-\alpha \rho \int_{0}^{1} \frac{\mathrm{d} x}{x^{\alpha}}\right)+c_{-}\left(\int_{0}^{1} \frac{(1-x)^{\alpha \rho}-1}{x^{\alpha+1}} \mathrm{~d} x+\alpha \rho \int_{0}^{1} \frac{\mathrm{d} x}{x^{\alpha}}\right) \\
& =c_{+} \int_{0}^{\infty} \frac{(1+x)^{\alpha \rho}-1}{x^{\alpha+1}} \mathrm{~d} x+c_{-} \int_{0}^{1} \frac{(1-x)^{\alpha \rho}-1}{x^{\alpha+1}} \mathrm{~d} x-\alpha \rho \frac{c_{+}-c_{-}}{1-\alpha} \\
& =c_{+} \rho \int_{0}^{\infty}(1+x)^{\alpha \rho-1} x^{-\alpha} \mathrm{d} x+c_{-}\left(\frac{1}{\alpha}-\rho \int_{0}^{1}(1-x)^{\alpha \rho-1} x^{-\alpha} \mathrm{d} x\right)-\alpha \rho \frac{c_{+}-c_{-}}{1-\alpha} \\
& =c_{+} \rho B(\alpha(1-\rho), 1-\alpha)+c_{-}\left(\alpha^{-1}-\rho B(\alpha \rho, 1-\alpha)\right)-a \alpha \rho .
\end{aligned}
$$


In the last equality, $B(x, y)=\Gamma(x+y)^{-1} \Gamma(x) \Gamma(y)$. Now let us check that

$$
c_{+} B(\alpha(1-\rho), 1-\alpha)=c_{-} B(\alpha \rho, 1-\alpha) .
$$

This is equivalent to

$$
c_{+} \Gamma(\alpha \rho) \Gamma(1-\alpha \rho)=c_{-} \Gamma(\alpha \bar{\rho}) \Gamma(1-\alpha \bar{\rho}),
$$

where $\bar{\rho}=1-\rho$. From the identity $\Gamma(x) \Gamma(1-x)=\pi / \sin (\pi x), 0<x<1$, we deduce that (20) is equivalent to

$$
\beta=\frac{\sin (\pi \alpha \bar{\rho})-\sin (\pi \alpha \rho)}{\sin (\pi \alpha \bar{\rho})+\sin (\pi \alpha \rho)}=\frac{\tan [(1-2 \rho) \pi \alpha / 2]}{\tan (\pi \alpha / 2)},
$$

which is correct according to (7). By putting (20) and (19) together, we obtain

$$
a_{2}=c_{-} \alpha^{-1}-a \alpha \rho \text {. }
$$

For $\alpha=1$, we suppose that $\rho=\frac{1}{2}$, so the computation is easy and also gives (21). For $\alpha \in(1,2)$, we present the computation of the first term on the right-hand side of (18) only:

$$
\begin{aligned}
\int_{0}^{1} & \frac{(1+x)^{\alpha \rho}-1-\alpha \rho x}{x^{\alpha+1}} \mathrm{~d} x+\int_{1}^{\infty} \frac{(1+x)^{\alpha \rho}-1}{x^{\alpha+1}} \mathrm{~d} x \\
& =\lim _{\varepsilon \downarrow 0} \int_{\varepsilon}^{\infty} \frac{(1+x)^{\alpha \rho}-1}{x^{\alpha+1}} \mathrm{~d} x-\alpha \rho \int_{\varepsilon}^{1} \frac{\mathrm{d} x}{x^{\alpha}} \\
& =\lim _{\varepsilon \downarrow 0} \frac{\rho}{1-\alpha} \varepsilon^{1-\alpha}+\rho \int_{\varepsilon}^{\infty} \frac{(1+x)^{\alpha \rho-1}-1}{x^{\alpha}} \mathrm{d} x-\frac{\alpha \rho}{1-\alpha} \\
& =\lim _{\varepsilon \downarrow 0} \frac{\rho}{1-\alpha} \varepsilon^{1-\alpha}-\frac{\rho(\alpha \rho-1)}{1-\alpha} \int_{\varepsilon}^{\infty} \frac{(1+x)^{\alpha \rho-2}-1}{x^{\alpha}} \mathrm{d} x-\frac{\alpha \rho}{1-\alpha} \\
& =\rho \frac{\alpha \rho-1}{\alpha-1} B(\alpha(1-\rho), 2-\alpha)-\frac{\alpha \rho}{1-\alpha} .
\end{aligned}
$$

The other term is computed similarly, and leads to

$$
a_{2}=c_{+} \rho \frac{\alpha \rho-1}{\alpha-1} B(\alpha(1-\rho), 2-\alpha)+c_{-}\left(\alpha^{-1}-\rho \frac{\alpha \rho-1}{\alpha-1} B(\alpha \rho, 2-\alpha)\right)-a \alpha \rho,
$$

which can also be reduced to (21).

Identity (21) shows that the killing rate in the expression, (16), for the generator $\mathcal{K}^{\uparrow}$ vanishes, which is in accordance with the fact that $\left(X, \mathbb{P}_{x}^{\uparrow}\right)$ belongs to the class $\mathcal{C}_{1}$. In conclusion, the expression of the infinitesimal generator of the process $\left(X, \mathbb{P}_{x}^{\uparrow}\right)$ is given by

$$
\begin{aligned}
\mathcal{K}^{\uparrow} f(x)= & \frac{1}{x^{\alpha}} \int_{\mathbb{R}^{+}}\left[f(u x)-f(x)-x f^{\prime}(x)(u-1) \mathbf{1}_{\{|u-1|<1\}}\right] v^{\uparrow}(u) \mathrm{d} u \\
& +\left(a+a_{1}\right) x^{1-\alpha} f^{\prime}(x) .
\end{aligned}
$$

As we did for $\left(X, \mathbb{P}_{x}\right)$ in the previous subsection, we may now apply Theorem 1 together with (in this case) (16) to compute the characteristics of the underlying Lévy process in the Lamperti representation of $\left(X, \mathbb{P}_{x}^{\uparrow}\right)$. 
Corollary 2. Let $\xi^{\uparrow}$ be the Lévy process in the Lamperti representation (1) of the PSSMP $\left(X, \mathbb{P}_{x}^{\uparrow}\right)$ defined in (13). The infinitesimal generator, $\mathcal{L}^{\uparrow}$, of $\xi^{\uparrow}$, with domain $\mathfrak{D}_{\mathcal{L}^{\uparrow}}$, is given by

$$
\mathcal{L}^{\uparrow} f(x)=a^{\uparrow} f^{\prime}(x)+\int_{\mathbb{R}}\left[f(x+y)-f(x)-f^{\prime}(x)\left(\mathrm{e}^{y}-1\right) \mathbf{1}_{\left\{\left|\mathrm{e}^{y}-1\right|<1\right\}}\right] \pi^{\uparrow}(y) \mathrm{d} y
$$

for any $f \in \mathfrak{D}_{\mathcal{L}^{\uparrow}}$ and $x>0$, where $\pi^{\uparrow}(y)=\mathrm{e}^{(\alpha \rho+1) y} v\left(\mathrm{e}^{y}-1\right), y \in \mathbb{R}$, and $a^{\uparrow}=a+a_{1}$, the constant $a_{1}$ being as defined in (17). Equivalently, the characteristic exponent of $\xi^{\uparrow}$ is given by

$$
\Phi^{\uparrow}(\lambda)=\mathrm{i} a^{\uparrow} \lambda+\int_{\mathbb{R}}\left[\mathrm{e}^{i \lambda y}-1-\mathrm{i} \lambda\left(\mathrm{e}^{y}-1\right) \mathbf{1}_{\left\{\left|\mathrm{e}^{y}-1\right|<1\right\}}\right] \pi^{\uparrow}(y) \mathrm{d} y .
$$

It follows from (14) that the underlying Lévy process $\xi^{\uparrow}$ drifts to $\infty$. The process being integrable, this means in particular that $0<\mathbb{E}\left[\xi_{1}^{\uparrow}\right]=-\mathrm{i}\left(\Phi^{\uparrow}\right)^{\prime}(0)<\infty$.

\subsection{The process conditioned to hit 0 continuously}

Let $W$ be an integer-valued random walk whose law is in the domain of attraction of the stable law $\left(X_{1}, \mathrm{P}_{0}\right)$. For $y \in \mathbb{Z} \backslash\{0\}$, define the law of the chain $W_{y}^{\searrow}$ as that of the random walk $W_{y}$ starting from $y$ and conditioned to hit 0 , as follows, where $\tau_{(-\infty, 0]}^{\searrow}:=\inf \left\{n: W_{n}^{\searrow} \leq 0\right\}$ and $\tau_{(-\infty, 0]}:=\inf \left\{n: W_{n} \leq 0\right\}:$

$$
\begin{aligned}
\left(W_{y}^{\searrow}(n), 0 \leq n \leq \tau_{(-\infty, 0]}^{\searrow}\right) & \stackrel{\mathrm{D}}{=}\left[\left(W_{y}(n), 0 \leq n \leq \tau_{(-\infty, 0]}\right) \mid W_{y}\left(\tau_{(-\infty, 0]}\right)=0\right], \\
\left(W_{y}^{\searrow}(n), n \geq \tau_{(-\infty, 0]}^{\searrow}\right) & \equiv 0 .
\end{aligned}
$$

(Here, 'D ' denotes equality in law.) It has recently been proved, in [5], that as $n$ tends to $\infty$ the rescaled linear interpolation of $W_{y}^{\searrow}$, i.e.

$$
\left(n^{-1 / \alpha} W_{\left\lfloor n^{1 / \alpha} x\right\rfloor}^{\searrow}(\lfloor n t\rfloor), t \geq 0\right),
$$

converges in law in the Skorokhod space to a Markov process which we will here call the Lévy process $\left(X, \mathrm{P}_{x}\right)$ conditioned to hit 0 continuously. Again, this process may be defined more formally as an $h$-transform of the killed process $\left(X, \mathbb{P}_{x}\right)$ introduced in Subsection 3.1. In this case, the positive harmonic function related to $\left(X, \mathbb{P}_{x}\right)$ is $g(x)=x^{\alpha \rho-1}$ and, for $x>0$, the law, $\mathbb{P}_{x}$, of the conditioned process is defined by

$$
\begin{aligned}
& \mathbb{P}_{x}^{\searrow}(A, t<S)=g(x)^{-1} \mathrm{E}_{x}\left[g\left(X_{t}\right) \mathbf{1}_{A} \mathbf{1}_{\{t<T\}}\right], \\
& \mathbb{P}_{x}^{\searrow}\left(X_{t}=0 \text { for all } t \geq S\right)=1,
\end{aligned}
$$

for all $x>0, t \geq 0$, and $A \in \mathcal{F}_{t}$. It was proved in [7] that the process $\left(X, \mathbb{P}_{x}^{\searrow}\right)$ reaches 0 continuously (perhaps by an accumulation of negative jumps if $\left(X, \mathrm{P}_{X}\right)$ has negative jumps), i.e.,

$$
\mathbb{P}_{x}^{\searrow}\left(X_{S-}=0\right)=1
$$

hence, $\left(X, \mathbb{P}_{x}^{\searrow}\right)$ is a PSSMP which belongs to the class $\mathcal{C}_{2}$. The infinitesimal generator of $\left(X, \mathbb{P}_{x}^{\searrow}\right)$ is given by

$$
\mathcal{K}^{\searrow} f(x)=\frac{1}{g(x)} \mathcal{K}(g f)(x), \quad x>0, f \in \mathfrak{D}_{\mathcal{K} \backslash} .
$$


Trivially, when there are no negative jumps (i.e. $\alpha \rho=1), g \equiv 1$ and the processes $\left(X, \mathbb{P}_{x}\right)$ and $\left(X, \mathbb{P}_{x}^{\searrow}\right)$ are the same. Calculations analogous to those in Subsection 3.2, replacing $\alpha \rho$ by $\alpha \rho-1$, lead to

$$
\begin{aligned}
\mathcal{K}^{\searrow} f(x)= & \frac{1}{x^{\alpha}} \int_{\mathbb{R}^{+}}\left[f(u x)-f(x)-x f^{\prime}(x)(u-1) \mathbf{1}_{\{|u-1|<1\}}\right] \nu \searrow(u) \mathrm{d} u \\
& +\left(a+a_{3}\right) x^{1-\alpha} f^{\prime}(x)+\left(a(\alpha \rho-1)+a_{4}-c_{-} \alpha^{-1}\right) x^{-\alpha} f(x),
\end{aligned}
$$

where

$$
\begin{aligned}
v \searrow(u)= & u^{\alpha \rho-1} v(u-1), \\
a_{3}= & c_{+} \int_{0}^{1} \frac{(1+x)^{\alpha \rho-1}-1}{x^{\alpha}} \mathrm{d} x+c_{-} \int_{0}^{1} \frac{(1-x)^{\alpha \rho-1}-1}{x^{\alpha}} \mathrm{d} x, \\
a_{4}= & c_{+}\left(\int_{0}^{1} \frac{(1+x)^{\alpha \rho-1}-1-(\alpha \rho-1) x}{x^{\alpha+1}} \mathrm{~d} x+\int_{1}^{\infty} \frac{(1+x)^{\alpha \rho-1}-1}{x^{\alpha+1}} \mathrm{~d} x\right) \\
& +c_{-} \int_{0}^{1} \frac{(1-x)^{\alpha \rho-1}-1+(\alpha \rho-1) x}{x^{\alpha+1}} \mathrm{~d} x .
\end{aligned}
$$

Again, since $\left(X, \mathbb{P}_{x}^{\searrow}\right)$ belongs to $\mathcal{C}_{2}$, the killing rate, $a \alpha \rho+a_{4}-c_{-} \alpha^{-1}$, of its generator vanishes. This can be checked through calculations analogous to those in Subsection 3.2. The expression for the infinitesimal generator of $\left(X, \mathbb{P}_{x}^{\searrow}\right)$ is found to be

$$
\begin{aligned}
\mathcal{K}^{\searrow} f(x)= & \frac{1}{x^{\alpha}} \int_{\mathbb{R}^{+}}\left[f(u x)-f(x)-x f^{\prime}(x)(u-1) \mathbf{1}_{\{|u-1|<1\}}\right] v \searrow(u) \mathrm{d} u \\
& +\left(a+a_{3}\right) x^{1-\alpha} f^{\prime}(x) .
\end{aligned}
$$

As in the previous subsections, we may now compute the characteristics of the underlying Lévy process in the Lamperti representation of $\left(X, \mathbb{P}_{x}^{\searrow}\right)$.

Corollary 3. Let $\xi \searrow$ be the Lévy process in the Lamperti representation (1) of the PSSMP $\left(X, \mathbb{P}_{x}^{\searrow}\right)$ defined in (22). The infinitesimal generator, $\mathcal{L}^{\searrow}$, of $\xi \searrow$, with domain $\mathfrak{D}_{\mathscr{L}} \downarrow$, is given by

$$
\mathcal{L}^{\searrow} f(x)=a^{\searrow} f^{\prime}(x)+\int_{\mathbb{R}}\left[f(x+y)-f(x)-f^{\prime}(x)\left(\mathrm{e}^{y}-1\right) \mathbf{1}_{\left\{\left|\mathrm{e}^{y}-1\right|<1\right\}}\right] \pi^{\searrow}(y) \mathrm{d} y
$$

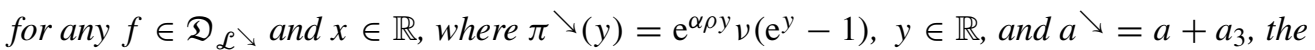
constant $a_{3}$ being as defined in (23). Equivalently, the characteristic exponent of $\xi \searrow$ is given by

$$
\Phi \searrow(\lambda)=\mathrm{i} a \searrow \lambda+\int_{\mathbb{R}}\left[\mathrm{e}^{\mathrm{i} \lambda y}-1-\mathrm{i} \lambda\left(\mathrm{e}^{y}-1\right) \mathbf{1}_{\left\{\left|\mathrm{e}^{y}-1\right|<1\right\}}\right] \pi \searrow(y) \mathrm{d} y .
$$

As noted above, the process $\left(X, \mathbb{P}_{x}^{\searrow}\right)$ belongs to the class $\mathcal{C}_{2}$ and, therefore, the underlying Lévy process $\xi \searrow$ drifts to $-\infty$; in particular, since this process is also integrable, we have $-\infty<\mathbb{E}\left[\xi_{1}^{\searrow}\right]=-\mathrm{i}(\Phi \searrow)^{\prime}(0)<0$.

\section{The minimum of $\xi$ up to an independent exponential time}

Using the same notation for $\left(X, \mathbb{P}_{x}\right),\left(X, \mathrm{P}_{x}\right)$, and $\xi$ as in Subsection 3.1, we here suppose that $\left(X, \mathrm{P}_{x}\right)$ has negative jumps, i.e. $\alpha \rho<1$ (which is equivalent to $c_{-}>0$ ). Recall that 
the characteristics of $\xi$ were computed in Corollary 1. The first result of this section is the computation of an explicit form of the law of the overall minimum of $\xi$. Since $\xi$ has a finite lifetime, it has the same law as a Lévy process, say $\tilde{\xi}$, that has an infinite lifetime and is killed at an independent exponential time with parameter $c_{-} \alpha^{-1}$. This allows us to show how the Lamperti representation, together with classical results on undershoots of subordinators, leads to the computation of the law of the minimum of $\tilde{\xi}$ up to an independent exponential time with parameter $c_{-} \alpha^{-1}$. The latter random variable is known as the spatial Wiener-Hopf factor of the Lévy process $\tilde{\xi}$; see [8].

Set $\underline{X}=\inf _{s \leq S} X_{s}$ and $\xi=\inf _{s \leq \zeta} \xi_{s}$, where we recall from the introduction that $S=$ $\inf \left\{t: X_{t}=0\right\}$ and $\zeta \equiv \zeta(\xi)$ is the lifetime of $\xi$. Then, on the one hand, from the Lamperti representation (1), under $\mathbb{P}_{x}$ the variables $\underline{X}$ and $\underline{\xi}$ are related as follows:

$$
\underline{X}=x \exp (\underline{\xi}) \quad \mathbb{P}_{x} \text {-a.s. }
$$

On the other hand, let $H$ be the downward ladder height process associated with $\left(X, \mathrm{P}_{0}\right)$, i.e. $H_{t}=-X_{\eta_{t}}$, where $\eta$ is the right-continuous inverse of the local time at 0 of the process $\left(X, \mathrm{P}_{0}\right)$ reflected at its minimum, $\left(X-\underline{X}, \mathrm{P}_{0}\right)$. We refer the reader to $[1$, Chapter $\mathrm{VI}]$, for a definition of ladder height processes. It is easy to verify the following identity, where $v(x)=$ $\inf \left\{t: H_{t}>x\right\}$ :

$$
\underline{X}=x-H_{v(x)-} \quad \mathbb{P}_{x} \text {-a.s. }
$$

In other words, $\underline{X}$ corresponds to the so-called undershoot of the subordinator $H$ at level $x$. Since $H$ is a stable subordinator with index $\alpha \rho$, the law of $\underline{X}$, and, hence, that of $\underline{\xi}$, can be computed explicitly as shown in the next proposition. In the sequel, $\mathbb{P}$ will be a reference probability measure under which $\xi$ and $H$ have the laws described above.

Proposition 1. Recall that $\rho=\mathrm{P}_{0}\left(X_{1}<0\right)$ and let $\xi$ be the Lévy process whose law is described in Corollary 1. For any $\lambda>0$,

$$
\mathbb{E}[\exp (\lambda \underline{\xi})]=\frac{\Gamma(\lambda+1-\alpha \rho)}{\Gamma(\lambda+1) \Gamma(1-\alpha \rho)} .
$$

In other words, $\exp (\underline{\xi})$ is a Beta variable with parameters $\alpha \rho$ and $1-\alpha \rho$, i.e. $\exp (\xi)$ has density function

$$
\mathbb{P}(\exp (\underline{\xi}) \in \mathrm{d} t)=B(\alpha \rho, 1-\alpha \rho)^{-1} t^{\alpha \rho-1}(1-t)^{-\alpha \rho} \mathbf{1}_{\{t \in[0,1]\}} \mathrm{d} t .
$$

Proof. Recall that the Lévy measure, $\theta(\mathrm{d} y)$, of $H$ and its potential measure, $U(\mathrm{~d} y)$, are given by

$$
\theta(\mathrm{d} y)=c_{1} y^{-(\alpha \rho+1)} \mathbf{1}_{\{y>0\}} \mathrm{d} y \quad \text { and } \quad \int_{0}^{\infty} \mathrm{e}^{-\lambda y} U(\mathrm{~d} y)=c_{2} \lambda^{-\alpha \rho}
$$

where $c_{1}$ and $c_{2}$ are positive constants. Then, from [1, Chapter III, Proposition 2],

$$
\mathbb{P}\left(H_{v(x)-} \in \mathrm{d} y\right)=\mathbf{1}_{\{y \in[0, x]\}} \int_{x}^{\infty} U(\mathrm{~d} y) \theta(\mathrm{d} z-y),
$$


from which we obtain, for all $\lambda \geq 0$ and $\mu \geq 0$,

$$
\begin{aligned}
\int_{0}^{\infty} \mathrm{e}^{-\mu x} \mathrm{E}\left[\mathrm{e}^{-\lambda\left(x-H_{\nu(x)-)}\right] \mathrm{d} x}\right. & =\int_{0}^{\infty} \mathrm{e}^{-(\lambda+\mu) x} \int_{0}^{x} \mathrm{e}^{\lambda y} \int_{x}^{\infty} U(\mathrm{~d} y) \theta(\mathrm{d} z-y) \mathrm{d} x \\
& =\int_{0}^{\infty} \mathrm{e}^{-(\lambda+\mu) x} \int_{0}^{x} \frac{c_{1}}{\alpha \rho} \mathrm{e}^{\lambda y}(x-y)^{-\alpha \rho} U(\mathrm{~d} y) \mathrm{d} x \\
& =\frac{c_{1} c_{2}(\lambda+\mu)^{\alpha \rho-1}}{\alpha \rho \mu^{\alpha \rho}} \Gamma(1-\alpha \rho) \\
& =\frac{(\lambda+\mu)^{\alpha \rho-1}}{\mu^{\alpha \rho}} .
\end{aligned}
$$

This means that if $\varsigma$ is exponentially distributed with parameter $\mu$ and independent of $H$, then $\varsigma-H_{v(\varsigma)-}$ is gamma distributed with parameters $\mu$ and $1-\alpha \rho$, i.e.

$$
\mathbb{E}\left[\mathrm{e}^{-\lambda\left(\varsigma-H_{\nu(\varsigma)-}\right)}\right]=\left(\frac{\mu}{\lambda+\mu}\right)^{1-\alpha \rho} .
$$

Recall that the moment of order $\lambda>0$ of the gamma law with parameters $\mu$ and $1-\alpha \rho$ is $\Gamma(\lambda+1-\alpha \rho) /\left(\mu^{\lambda} \Gamma(1-\alpha \rho)\right)$. Let $\gamma$ be a random variable with this law. Then, from (24) and (25), we have

$$
\mathbb{E}[\exp (\lambda \underline{\xi})]=\frac{\mathbb{E}\left[\gamma^{\lambda}\right]}{\mathbb{E}\left[\varsigma^{\lambda}\right]}=\frac{\Gamma(\lambda+1-\alpha \rho)}{\Gamma(\lambda+1) \Gamma(1-\alpha \rho)},
$$

which is the moment of order $\lambda$ of a Beta variable with parameters $\alpha \rho$ and $1-\alpha \rho$.

In view of the result of Proposition 1, we might be tempted to compute the law of the overall minimum, $\inf _{t \leq e(\mu)} \tilde{\xi}_{t}$, of the unkilled process $\tilde{\xi}$ before an independent exponential time of any parameter $\mu>0$. However, although the PSSMP which is obtained from $\left(\tilde{\xi}_{t}, t \leq e(\mu)\right)$ through the Lamperti representation is absolutely continuous with respect to $\left(X, \mathbb{P}_{x}\right)$, its law is not sufficiently explicit to apply the same arguments as in Proposition 1.

We can, nevertheless, apply the arguments used above to determine the law of the overall minimum of the Lévy process $\xi^{\uparrow}$ defined in Subsection 3.2. Indeed, as we observed there, $\xi^{\uparrow}$ drifts to $\infty$, as does the PSSMP $\left(X, \mathbb{P}_{x}^{\uparrow}\right)$, and from the Lamperti representation the relation

$$
\underline{X}=x \exp \left(\underline{\xi}^{\uparrow}\right) \quad \mathbb{P}_{x}^{\uparrow} \text {-a.s. }
$$

holds. Moreover, the law of $\left(\underline{X}, \mathbb{P}_{x}^{\uparrow}\right)$ is explicit and may be found in [7, Theorem 5]: for all $x>0$,

$$
\mathbb{P}_{x}^{\uparrow}(\underline{X} \leq y)=\frac{x^{\alpha \rho}-(x-y)^{\alpha \rho} \mathbf{1}_{\{y \leq x\}}}{x^{\alpha \rho}} .
$$

This allows us to state our final result.

Proposition 2. Let $\xi^{\uparrow}$ be the Lévy process whose law is described in Corollary 2. The law of the overall minimum, $\underline{\xi}^{\uparrow}$, of $\xi^{\uparrow}$ is given by

$$
\mathbb{P}\left(-\underline{\xi}^{\uparrow} \leq z\right)=\left(1-\mathrm{e}^{-z}\right)^{\alpha \rho} \mathbf{1}_{\{z \geq 0\}} .
$$

This result is closely related to risk theory and, in particular, provides the explicit form of the ruin probability at level $z \geq 0$, i.e. for this class of Lévy processes,

$$
\mathbb{P}\left(\text { there exists a } t \geq 0 \text { such that } z+\xi_{t}^{\uparrow} \leq 0\right)=\mathbb{P}\left(\underline{\xi}^{\uparrow} \leq-z\right)=1-\left(1-\mathrm{e}^{-z}\right)^{\alpha \rho}
$$

see the recent paper by Lewis and Mordecki [11]. 


\section{Acknowledgements}

This work was partly done during the visit of the second author to UNAM. He is very grateful to this university for its support. In addition, this research was partially supported by the project PAPIIT-IN120605. We thank Pat Fitzsimmons for fruitful discussions and valuable comments. We are also much indebted to an anonymous referee for his/her careful reading, which allowed us to shorten some proofs in the paper and improve the final version.

\section{References}

[1] Bertoin, J. (1996). Lévy Processes. Cambridge University Press.

[2] Bertoin, J. (2002). Self-similar fragmentations. Ann. Inst. H. Poincaré Prob. Statist. 38, 319-340.

[3] Bingham, N. H. (1973). Maxima of sums of random variables and suprema of stable processes. Z. Wahrscheinlichkeitsth. 26, 273-296.

[4] Bryn-Jones, A. And Doney, R. A. (2006). A functional limit theorem for random walk conditioned to stay non-negative. J. London Math. Soc. 74, 244-258.

[5] Caravenna, F. and Chaumont, L. (2006). Invariances principles for conditioned random walks. Preprint 1050, LPMA.

[6] Carmona, P., Petit, F. and Yor, M. (2001). Exponential functionals of Lévy processes. In Lévy Processes, Birkhäuser, Boston, MA, pp. 41-55.

[7] Chaumont, L. (1996). Conditionings and path decompositions for Lévy processes. Stoch. Process. Appl. 64, 39-54.

[8] Doney, R. A. (2005). Fluctuation theory for Lévy processes. To appear in École d'été de Probabilités de Saint-Flour.

[9] Duquesne, T. and Le Gall, J. F. (2002). Random Trees, Lévy Processes and Spatial Branching Processes (Astérisque 281). Société Mathématique de France, Paris.

[10] Lamperti, J. W. (1972). Semi-stable Markov processes. Z. Wahrscheinlichkeitsth. 22, 205-225.

[11] LewIS, A. AND MordecKI, E. (2005). Wiener-Hopf factorization for Lévy processes having negative jumps with rational transforms. Tech. Rep.

[12] Rivero, V. (2005). Recurrent extensions of self-similar Markov processes and Cramér's condition. Bernoulli 11, 471-509.

[13] Sato, K. I. (1999). Lévy Processes and Infinitely Divisible Distributions. Cambridge University Press.

[14] Zolotarev, V. M. (1986). One-Dimensional Stable Distributions (Transl. Math. Monogr. 65). American Mathematical Society, Providence, RI. 